\title{
Prognostic Significance of plasma albumin to fibrinogen ratio Associated Nomograms in Patients with breast invasive ductal carcinoma
}

\section{Lihua Zheng}

Hebei Medical University First Affiliated Hospital https://orcid.org/0000-0003-1445-0961

Feng Liu

Hebei Medical University First Affiliated Hospital

Yaheng Zhao

Hebei Medical University First Affiliated Hospital

Wei Li

Hebei Medical University First Affiliated Hospital

Yan Yang

Hebei Medical University First Affiliated Hospital

Hongsong Zhang

Hebei Medical University First Affiliated Hospital

Yunjiang Liu ( $D$ lyj818326@outlook.com )

https://orcid.org/0000-0001-7202-2004

Research article

Keywords: Breast invasive ductal carcinoma, Albumin to fibrinogen ratio(AFR), Prognostic nomogram

Posted Date: August 14th, 2019

DOI: https://doi.org/10.21203/rs.2.12803/v1

License: (c) (1) This work is licensed under a Creative Commons Attribution 4.0 International License.

Read Full License 


\section{Abstract}

Background: In the context of breast invasive ductal carcinoma, this research aims to retrospectively evaluate by preoperative plasma albumin to fibrinogen ratio (AFR) and forecast oncological outcome and recurrence. Methods: This reflective study included 230 patients who had surgical procedures at the Fourth Hospital of Hebei Medical University between January 2009 and April 2012 for the treatment of their non-metastatic breast invasive ductal carcinoma. We utilized an optimal value of preoperative plasma fibrinogen and albumin for the patient classification. Additionally, we used the Kaplan-Meier method to extensively evaluate progression-free and cancer-specific survival outcomes. The analysis of the linkages between the albumin to fibrinogen ratio and clinical outcomes was executed through the univariate and multivariate analyses. To construct nomograms and evaluate the survival outcomes, we used a variety of risk factors. We also verified the predictive accuracy through the Harrell's concordance index (C-index). Results : The association of plasma AFR with diminished disease-free survival (DFS) and overall survival (OS) is statistically significant. An independent prognostic indicator is the plasma AFR, as reported by the multivariate analysis, for DFS (HR $=1.346 ; 95 \% \mathrm{Cl}: 1.107-1.636 ; \mathrm{p}=0.03$ ) and overall survival (OS) (HR $=1.485 ; 95 \% \mathrm{Cl}: 1.106-1.993 ; p=0.008)$. Two prediction model of OS and DFS based on the AFR was developed. Conclusions : For patients with breast invasive ductal carcinoma, an independent prognostic factor for the outcomes of oncology patients is the elevated preoperative plasma AFR. For DFS and OS, the constructed nomogram demonstrated highly significant predictive accuracy.

\section{Background}

The most invasive cancer among women is breast cancer (BC), which is confirmed as the second leading cause of cancer-associated mortality. Taking into consideration the health burdens, high morbidity and mortality rates among women, this disease has become a global health concern. Recent reports have evaluated the prognostic value of the systemic inflammatory markers and its relevance to breast cancer, which include C-reactive protein[1], albumin[2], neutrophils[3], platelets [4], lymphocytes[5], and fibrinogen[6] as the single markers.

Hepatocytes produce mainly fibrinogen and albumin. As described, it is worth noting how both epithelial and tumor cells synthesize extrahepatically [7]. The main goal of this research study is to extensively evaluate AFR's predictive value in postoperative breast invasive ductal carcinoma to construct a nomogram that could form novel prognostic models. Eventually, the established nomogram led to the refinement of two novel nomogram-based DFS and OS.

\section{Methods And Materials}

Two hundred thirty women, who had not undergone any form of radiation therapy, systemic chemotherapy, or hormone therapy before their surgeries, participated in this study. These women had primary surgical procedures that occurred from between January 2009 to April 2012 at the Department of Breast Surgery in the Fourth Hospital of Hebei Medical University. 
We conducted follow-ups with all our patients until July 2016 or the date of their deaths. The $7^{\text {th }}$ American Joint Committee on Cancer comprehensively evaluated the pathologic TNM stage [8-9]. The expression of the tumor cells at $30 \%$ or higher correspondingly led to a high Ki67 index score. We conducted all-inclusive analyses for estrogen receptor (ER), progesterone receptor (PR), and HER2 that utilized the endorsed guidelines of the American Society of Clinical Oncology and College of American Pathologists [10-11]. The specimens with an HER2 IHC score of 2+ were removed in our study. Furthermore, according to the standardized guidelines, we executed applicable adjuvant treatments after the surgery. Most of the hormone receptor (HR) positive patients received the adjuvant hormone therapy for 5 years at least. But none of the patients received the HER2-targeted adjuvant therapy.

\section{Statistical analysis}

Empower Stats and R Project version 3.3.3 (http://www.r-project.org/) performed all of the statistical analyses and randomization. The median determined the cutoff value of AFR. We used the Kaplan-Meier curves to compare the overall survival rates and the Empower Stats' log-rank test to analyze accordingly. To ascertain the independent prognostic risk factors, the Cox proportional hazards regression model required the univariate and multivariate analyses along with the hazard ratios (HRs). The statistical significance of the difference was set at $P<0.05$. For DFS and OS, Empower Stats formulated two novel prognostic nomograms based on AFR. The R Project version formulated the predictive performance, which was also measured by the concordance index (C-index), calibration curve, and decision curve analysis (DCA).

\section{Results}

\section{List the clinical characteristics of the patients and the clinicopathological correlations}

The 230 female patients had a range of 27 to 81 years and a median age of 48 years. For the follow-up, the range was from 7 to 81 months and the median was 67 months. Means, standard deviations, and ranges of laboratory results for albumin, fibrinogen, and AFR were $41.29 \pm 3.39 \mathrm{~g} / \mathrm{d}$ (range, 31.4-50.6 $\mathrm{mg} / \mathrm{dL}$ ), $3.21 \pm 0.54 \mathrm{~g} / \mathrm{L}$ (range, $1.97-4.93 \mathrm{~g} / \mathrm{L}$ ), and $13.24 \pm 2.43$ (range, 7.92-19.40), respectively. The optimal values for AFR was13.1 by median(Table 1 ).

\section{Prognostic Value of AFR}

Adverse DFS and OS are associated with high levels of AFR expression. We conducted comprehensive investigations on the clinicopathological parameters for the prediction of DFS and OS using the univariate analysis with the Cox regression model. The univariate analysis presented results where the AFR is significantly related to DFS and OS. Then, for the multivariate Cox regression model, these variables with significant association were used. For patients with invasive ductal carcinoma, AFR continued to be influential and independent prognostic factors for both the DFS and OS models (Table 2).

\section{Prognostic value of AFR}


To give a reasonable stratification of OS, the patients were divided into 2 groups on the basis of median value of AFR : a low-risk group ( $A R<13.1, N=114$ ) and a high-risk group (AFR $\geq 13.1, N=116)$. The median OS of the low-risk group and high-risk group is 66.63 months and 62.36 months respectively. The median DFS of the low-risk group and high-risk group is 65.29 months and 59.97 months respectively. The DFS and OS were significantly different among the 2 subgroups $(P<0.05)$ (Figure $1 \mathrm{~A}$ and B).

\section{Novel prognostic nomogram for OS and DFS prediction}

Using the multivariate Cox regression model, we established two nomograms based on all the significant independent factors for DFS and OS to obtain the predictive values of DFS and OS for patients with AFR (Figure 2A and B).

On top of the scale, the points assigned to each variable are indicated.

The sum of these points is utilized to interpret the nomograms. In the lowest scale of the nomogram's Cindex, the sum could be converted to predicted 3- and 5-year DFS and OS for a patient. More specifically, the lowest scale of the nomogram is based on two values: first, the AFR predicted OS that has an accuracy value of 0.826 and second, the AFR predicted DFS that has an accuracy value of 0.834 . The calibration plots were illustrated for 3-year survival(Figure 3).

\section{The decision curve analysis for DFS and OS}

The $y$-axis on the decision curve analysis is the net benefit that was obtained through summing the true positives or benefits and subtracting the false positives or the harms. Additionally, the horizontal line represented the postulation that there will be no patient death while the straight line represented the postulation that all patients will die (Figure 4).

\section{Discussion}

This study highlights the significant and determining independent prognostic factor of the AFR in breast cancer. Few cancer researches have study AFR.

We confirmed that the association between the AFR levels with the DFS and OS of patients are independent by using the multivariate Cox regression analysis. Using univariable and subsequent multivariable analyses in this research, we identified WBC, TNM, lymphovascular invasion, nuclear grade, $\mathrm{ER} / \mathrm{PR}(+), \mathrm{P} 53$, and AFR as independent prognostic factors for OS. C-index of the nomogram based on AFR predicted OS with an accuracy of 0.882 , and, we identified WBC, TNM, lymphovascular invasion, nuclear grade, P53, Ki-67 and AFR as independent prognostic factors for DFS. C-index of the nomogram based on AFR predicted DFS with an accuracy of 0.922.

On one hand, when a predictor or model cannot discriminate, it has a value of 0.5 . On the other hand, a model that could perfectly discriminate has a value of 1.0 [13]. A model that could be considered good has a value of 0.70 to 0.80 while one that has an AUC of 0.80 to 0.90 is considered to have an excellent 
capability to discriminate [14-15]. With good discrimination and calibration, the nomograms demonstrated good performance. In the context of individual patients with breast invasive ductal carcinoma, this model could easily be identified as an accessible and straightforward tool for estimating OS and DFS.

To improve the prognostic predictive value for breast invasive ductal carcinoma patients, we constructed two nomograms that included AFR in this study. To provide better predictive values, these nomograms could be utilized in the context of the 3- and 5-year DFS and OS probability of a patient. Using calibration plots and the C-index, we formulated these two nomograms, which had an outstanding performance with good calibration. The C-index values had satisfactory values for DFS at 0.922 and OS at 0.884 .

Our reach was different from Ki-Tae Hwang's, who provided indicators that demonstrated the significant independent unfavorable prognosticator of breast cancer in preoperative AFR [16].

To conclude, this study reveals groundbreaking information that contrasts breast invasive ductal carcinoma with low AFR expression with those that have high AFR expression. The latter demonstrated higher risks of experiencing metastatic breast cancer and lower survival rates. Furthermore, an independent predictor of patient outcomes that our results highlight is the AFR expression in breast invasive ductal carcinoma. We constructed these two nomograms to systematically predict the probability of 3-and 5-year DFS and OS. Ultimately, the model opens the possibilities for personalized decision-making processes for adjuvant treatment, follow-up scheduling, and facilitation of clinician and patient counseling.

\section{Limitations}

The present study had several limitations. The sample size was still small. Although we creat nomograms, we do not validate them , future studies are needed to externally validate the proposed nomograms.

\section{Declarations}

\section{Limitations}

The present study had several limitations. The sample size was still small. Although we creat nomograms, we do not validate them , future studies are needed to externally validate the proposed nomograms.

\section{Authors' contributions}

Li-hua Zheng, writer the paper; Feng Liu, analysis and interpretation of data; Yan Yang, and Hongsong Zhang, Shuo Zhang, and Yaheng Zhao, collect the date; Yunjiang LIU,conception and design the paper;

\section{Funding source}


This study was funded by Hebei Provincial Health Planning Commission(20180234)

\section{Declaration of interest}

None of the authors has any potential conflicts to disclose.

\section{Availability of data and materials}

The datasets used and analyzed during the current study are available from the corresponding author on reasonable request.

\section{Ethical approval}

This article does not contain any studies with human participants or animals performed by any of the authors.

\section{Consent for publication}

Not applicable.

\section{Author details}

${ }^{1}$ Department of General surgery, the First Hospital of Hebei Medical University, Hebei Shijiazhuang 050011, China. ${ }^{2}$ Department of breast surgery, the Fourth Hospital of Hebei Medical University, Hebei Shijiazhuang 050011, China.

\section{References}

1. Shrotriya S, Walsh D, Bennani-Baiti N, Thomas S, Lorton C. C-reactive protein is an important biomarker for prognosis tumor recurrence and treatment response in adult solid tumors: a systematic review. PLoS One 2015;10:e0143080.

2. Gupta D, Lis CG. Pretreatment serum albumin as a predictor of cancer survival: a systematic review of the epidemiological literature. Nutr J 2010;9:69.

3. Uribe-Querol E, Rosales C. Neutrophils in cancer: two sides of the same coin. J Immunol Res 2015;2015:983698.

4. Menter DG, Tucker SC, Kopetz S, Sood AK, Crissman JD, Honn KV. Platelets and cancer: a casual or causal relationship: revisited. Cancer Metastasis Rev 2014;33:231-69.

5. Liang L, Zhu J, Jia H, Huang L, Li D, Li Q, et al. Predictive value of pretreatment lymphocyte count in stage II colorectal cancer and in high risk patients treated with adjuvant chemotherapy. Oncotarget 2016; 7:1014-28.

6. Perisanidis C, Psyrri A, Cohen EE, Engelmann J, Heinze G, Perisanidis B, et al. Prognostic role of pretreatment plasma fibrinogen in patients with solid tumors: a systematic review and meta- 
analysis. Cancer Treat Rev 2015;41:960-70.

7. Seebacher V, Polterauer S, Grimm C, et al.

The prognostic value of plasma fibrinogen levels in patients with endometrial cancer: a multicentre trial. Br J Cancer.2010;;102(6):952-956.

8. Elston C W, Ellis I O. Pathological prognostic factors in breast cancer. I. The value of histological grade in breast cancer: experience from a large study with long-term follow-up[J]. Histopathology, 1991, 19(5): 403-410.

9. Denkert C, Budczies J, von Minckwitz G, et al. Strategies for developing Ki67 as a useful biomarker in breast cancer[J]. The Breast, 2015, 24: S67-S72.

10. Hammond ME, Hayes DF, Dowsett M, et al. American Society of Clinical Oncology/College of American Pathologists guideline recommendations for immunohistochemical testing of estrogen and progesterone receptors in breast cancer (unabridged version)[J]. Arch Pathol Lab Med.2010,134(7):e48-72.

11. Wolff A C, Hammond M E H, Hicks D G, et al. Recommendations for human epidermal growth factor receptor 2 testing in breast cancer: American Society of Clinical Oncology/College of American Pathologists clinical practice guideline update[J]. Arch Pathol Lab Med, 2013, 138(2): 241-256.

12. Weiser MR, Gonen M, Chou JF, Kattan MW, and Schrag D (2011). Predicting survival after curative colectomy for cancer: individualizing colon cancer staging[J]. J Clin Oncol 29, 4796-4802.

13. Harrell FE Jr, Lee KL, Califf RM,et al. Regression modelling strategies for improved prognostic prediction.Stat Med[J]. $1984 ; 3(2): 143-152$.

14. Klar M, Jochmann A, Foeldi M, et al. The MSKCC nomogram for prediction the likelihood of nonsentinel node involvement in a German breast cancer population[J]. Breast Cancer Res Treat 2008; 112:523-531.

15. Hanley JA, McNeil BJ. A method of comparing the areas under receiver operating characteristic curves derived from the same cases[J]. Radiology 1983; 148:839-843.

16. Hwang KT, Chung JK, Roh EY, et al. Prognostic Influence of Preoperative Fibrinogen to Albumin Ratio for Breast Cancer. J Breast Cancer[J].2017;20(3):254-263.

\section{Tables}

Table 1. Clinical Characteristics of Patients 


\begin{tabular}{|c|c|c|c|}
\hline AFR & $<13.1$ (Low) & $\geq 13.1$ (High) & $P$ \\
\hline $\mathrm{N}$ & 114 & 116 & \\
\hline OS & $66.63 \pm 11.04$ & $62.36 \pm 15.07$ & 0.015 \\
\hline DFS & $65.29 \pm 13.74$ & $59.97 \pm 17.19$ & 0.010 \\
\hline AFR & $11.30 \pm 1.34$ & $15.13 \pm 1.63$ & $<0.001$ \\
\hline $\operatorname{Albumin}(\mathrm{g} / \mathrm{L})$ & $39.89 \pm 2.88$ & $42.67 \pm 3.28$ & $<0.001$ \\
\hline Fibrinogen(g/L) & $3.57 \pm 0.46$ & $2.85 \pm 0.34$ & $<0.001$ \\
\hline Age & $52.67 \pm 11.69$ & $48.52 \pm 10.59$ & 0.005 \\
\hline WBC & $6.43 \pm 1.49$ & $5.54 \pm 1.44$ & $<0.001$ \\
\hline $\mathrm{RBC}$ & $4.38 \pm 0.38$ & $4.28 \pm 0.43$ & 0.059 \\
\hline PLT & $246.83 \pm 64.52$ & $225.40 \pm 66.98$ & 0.014 \\
\hline HGB & $130.24 \pm 10.55$ & $128.63 \pm 16.27$ & 0.374 \\
\hline TNM & & & 0.176 \\
\hline 1 & $38(33.33 \%)$ & $32(27.59 \%)$ & \\
\hline 2 & $64(56.14 \%)$ & $62(53.45 \%)$ & \\
\hline 3 & $12(10.53 \%)$ & $22(18.97 \%)$ & \\
\hline Lymphovascular invasion & & & 0.631 \\
\hline No & $80(70.18 \%)$ & $78(67.24 \%)$ & \\
\hline Yes & $34(29.82 \%)$ & $38(32.75 \%)$ & \\
\hline Nuclear grade & & & $<0.001$ \\
\hline 1 & $12(10.53 \%)$ & $36(31.03 \%)$ & \\
\hline 2 & $82(71.93 \%)$ & $64(55.17 \%)$ & \\
\hline 3 & $20(17.54 \%)$ & $16(13.79 \%)$ & \\
\hline ER & & & 0.238 \\
\hline negative & $56(49.12 \%)$ & $48(41.38 \%)$ & \\
\hline positive & $58(50.88 \%)$ & $68(58.62 \%)$ & \\
\hline $\mathrm{PR}$ & & & 0.238 \\
\hline negative & $56(49.12 \%)$ & $48(41.38 \%)$ & \\
\hline positive & $58(50.88 \%)$ & $68(58.62 \%)$ & \\
\hline Her-2 & & & 0.143 \\
\hline negative & 66 (57.89\%) & 78 (67.24\%) & \\
\hline positive & $48(42.11 \%)$ & 38 (32.76\%) & \\
\hline P53 & & & 0.061 \\
\hline$\square 25 \%$ & $34(29.82 \%)$ & 18 (15.79\%) & \\
\hline$\geq 25 \% \square 50 \%$ & 38 (33.3333\%) & $50(43.86 \%)$ & \\
\hline$\geq 50 \% \square 75 \%$ & $22(19.30 \%)$ & $28(24.56 \%)$ & \\
\hline$\geq 75 \%$ & $20(17.54 \%)$ & $18(15.79 \%)$ & \\
\hline KI-67 & & & 0.133 \\
\hline$\leq 30 \%$ & $42(36.84 \%)$ & $32(27.59 \%)$ & \\
\hline$\square 30 \%$ & $72(63.16 \%)$ & $84(72.41 \%)$ & \\
\hline
\end{tabular}

Table 2. Univariate Analysis of OS and DFS 


\begin{tabular}{|c|c|c|c|c|}
\hline \multirow[t]{2}{*}{ Variables } & \multicolumn{2}{|l|}{ Overall Survival } & \multicolumn{2}{|c|}{ Disease-Free Survival } \\
\hline & HR $(95 \%$ CI) & $P$ value & HR $(95 \%$ CI) & $P$ value \\
\hline AFR & $1.0577(0.8942,1.2510)$ & 0.512531 & $1.0767(0.9449,1.2269)$ & 0.267470 \\
\hline Albumin & $1.0781(0.9416,1.2343)$ & 0.276206 & $1.0880(0.9770,1.2116)$ & 0.124550 \\
\hline Fibrinogen $\llbracket \mathrm{g} / \mathrm{L} \square$ & $0.8104(0.3693,1.7786)$ & 0.600174 & $0.7774(0.4203,1.4381)$ & 0.422419 \\
\hline Age & $0.9723(0.9328,1.0134)$ & 0.183509 & $0.9958(0.9665,1.0260)$ & 0.784089 \\
\hline WBC & $1.2066(0.9328,1.5608)$ & 0.152696 & $1.0211(0.8241,1.2652)$ & 0.848340 \\
\hline $\mathrm{RBC}$ & $3.8652(1.3662,10.9357)$ & 0.010834 & $2.3808(1.0097,5.6137)$ & 0.047471 \\
\hline HGB & $1.0341(0.9937,1.0761)$ & 0.099474 & $1.0001(0.9768,1.0240)$ & 0.990828 \\
\hline PLT & $0.9973(0.9911,1.0036)$ & 0.403274 & $0.9981(0.9933,1.0031)$ & 0.458423 \\
\hline \multicolumn{5}{|l|}{ TNM stage } \\
\hline 1 & 1.0 & & 1.0 & \\
\hline 2 & $4.6951(1.0794,20.4213)$ & 0.039216 & $3.6853(1.2785,10.6229)$ & 0.015743 \\
\hline 3 & $4.4155(0.8086,24.1130)$ & 0.086407 & $4.4888(1.3515,14.9097)$ & 0.014216 \\
\hline Tumor size & $1.0570(0.7980,1.4001)$ & 0.699184 & $1.1025(0.9014,1.3484)$ & 0.342169 \\
\hline \multicolumn{5}{|c|}{ Lymphovascular invasion } \\
\hline No & 1.0 & & 1.0 & \\
\hline Yes & $4.1391(1.7359,9.8695)$ & 0.001355 & $5.0933(2.5457,10.1904)$ & 0.000004 \\
\hline \multicolumn{5}{|l|}{ Nuclear grade } \\
\hline 1 & 1.0 & & 1.0 & \\
\hline 2 & $1.9486(0.4359,8.7109) 0.382583$ & & $3.4008(0.7949,14.5503)$ & 0.098852 \\
\hline 3 & $5.7902(1.2290,27.2790)$ & 0.026366 & $11.0623(2.5128,48.7007)$ & 0.001481 \\
\hline \multicolumn{5}{|l|}{$\mathrm{ER} / \mathrm{PR}$} \\
\hline Negative & 1.0 & & 1.0 & \\
\hline Positive & $0.2919(0.1142,0.7461)$ & 0.010124 & $0.3799(0.1899,0.7599)$ & 0.006215 \\
\hline \multicolumn{5}{|l|}{ Her-2 status } \\
\hline Negative & 1.0 & & 1.0 & \\
\hline Positive & $1.9241(0.8278,4.4723)$ & 0.128308 & $1.0983(0.5619,2.1467)$ & 0.783994 \\
\hline \multicolumn{5}{|l|}{ P53 } \\
\hline$\square 25 \%$ & 1.0 & & 1.0 & \\
\hline$\geq 25 \% \square 50 \%$ & $0.8807(0.2484,3.1221)$ & 0.844056 & $0.7106(0.2804,1.8005)$ & 0.471323 \\
\hline$\geq 50 \% \square 75 \%$ & $0.0000(0.0000, \mathrm{Inf})$ & 0.997645 & $0.2413(0.0512,1.1364)$ & 0.072132 \\
\hline$\geq 75 \%$ & $3.9070(1.2249,12.4616)$ & 0.021290 & $2.9852(1.2513,7.1218)$ & 0.013689 \\
\hline \multicolumn{5}{|l|}{ Ki67 } \\
\hline$\leq 30 \%$ & 1.0 & & 1.0 & \\
\hline$\square 30 \%$ & inf. $(0.0000, \mathrm{Inf})$ & 0.997195 & $9.1140(2.1890,37.9462)$ & 0.002393 \\
\hline
\end{tabular}

Table 3. Multivariable Cox Regression Analysis of OS and DFS 


\begin{tabular}{|c|c|c|c|c|}
\hline \multirow[t]{2}{*}{ Variables } & \multicolumn{2}{|l|}{ Overall Survival } & \multicolumn{2}{|c|}{ Disease-Free Survival } \\
\hline & HR $(95 \%$ CI) & $P$ value & HR $(95 \% \mathrm{CI})$ & $P$ value \\
\hline AFR & $1.3469(1.0697,1.6959)$ & 0.011312 & $1.3458(1.1072,1.6358)$ & 0.002852 \\
\hline WBC & $2.0854(1.3328,3.2628)$ & 0.001291 & & \\
\hline \multicolumn{5}{|l|}{ TNM } \\
\hline 1 & Reference & & Reference & \\
\hline 2 & $8.6919(1.6110,46.8952)$ & 0.011919 & $5.6041(1.8594,16.8908)$ & 0.002199 \\
\hline 3 & $2.6788(0.2708,26.5006)$ & 0.399396 & $3.6738(0.8561,15.7656)$ & 0.079959 \\
\hline \multicolumn{5}{|c|}{ Lymphovascular invasion } \\
\hline No & Reference & & Reference & \\
\hline Yes & $21.6410(4.8885,95.8034)$ & 0.000051 & $11.3356(4.6016,27.9238)$ & $<0.000001$ \\
\hline \multicolumn{5}{|l|}{ Nuclear grade } \\
\hline 1 & Reference & & Reference & \\
\hline 2 & $1.5753(0.1917,12.9430)$ & 0.672347 & $4.4498(0.7487,26.4470)$ & 0.100652 \\
\hline 3 & 23.4055 (2.4567, 222.9884) & 0.006115 & $25.3146(3.4831,183.9835)$ & 0.001407 \\
\hline \multicolumn{5}{|l|}{$\mathrm{ER} / \mathrm{PR}(+)$} \\
\hline Negative & Reference & & Reference & \\
\hline Positive & $0.1242(0.0345,0.4469)$ & 0.001410 & $0.5208(0.1943,1.3958)$ & 0.194605 \\
\hline \multicolumn{5}{|l|}{ P53 } \\
\hline$\square 25 \%$ & Reference & & Reference & \\
\hline$\geq 25 \% \square 50 \%$ & $1.5797(0.2837,8.7974)$ & 0.601763 & $0.3432(0.1003,1.1743)$ & 0.088372 \\
\hline$\geq 50 \% \square 75 \%$ & 0.0000 (0.0000, Inf) & 0.997943 & $0.0511(0.0085,0.3080)$ & 0.001178 \\
\hline$\geq 75 \%$ & $15.6747(3.1987,76.8116)$ & 0.000689 & $6.2352(2.1124,18.4047)$ & 0.000919 \\
\hline \multicolumn{5}{|l|}{ Ki-67 } \\
\hline$\leq 30 \%$ & & & Reference & \\
\hline$\square 30 \%$ & & & $7.9344(1.4828,42.4568)$ & 0.015507 \\
\hline
\end{tabular}

\section{Figures}




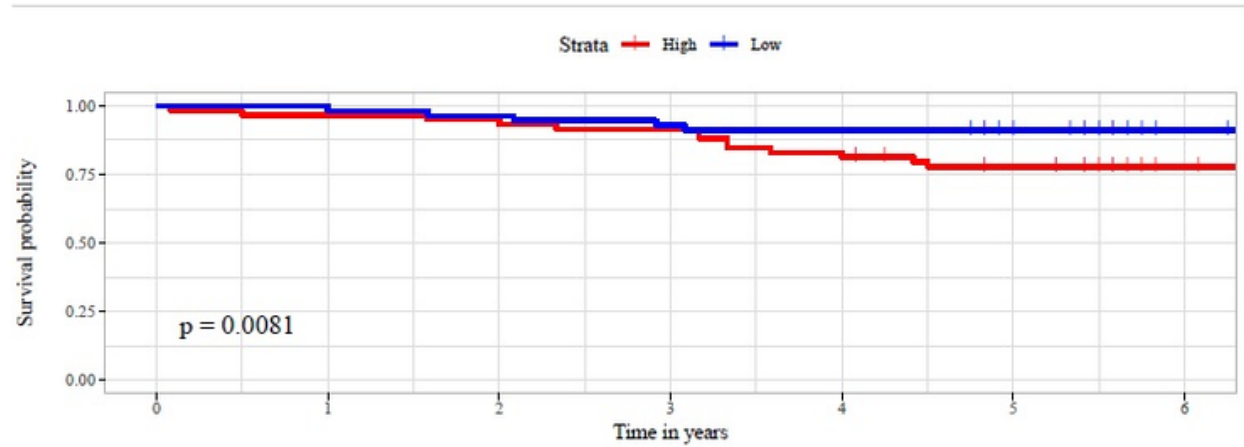

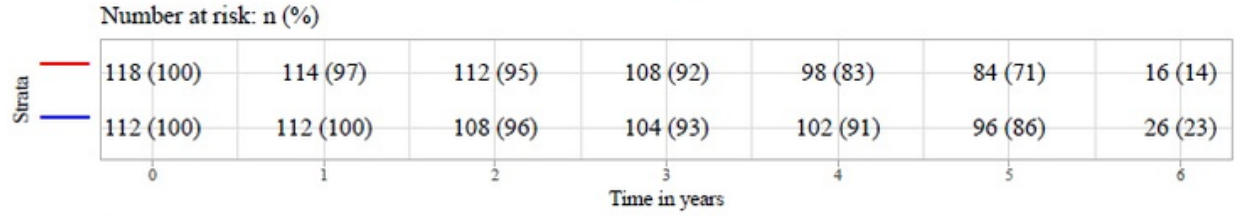
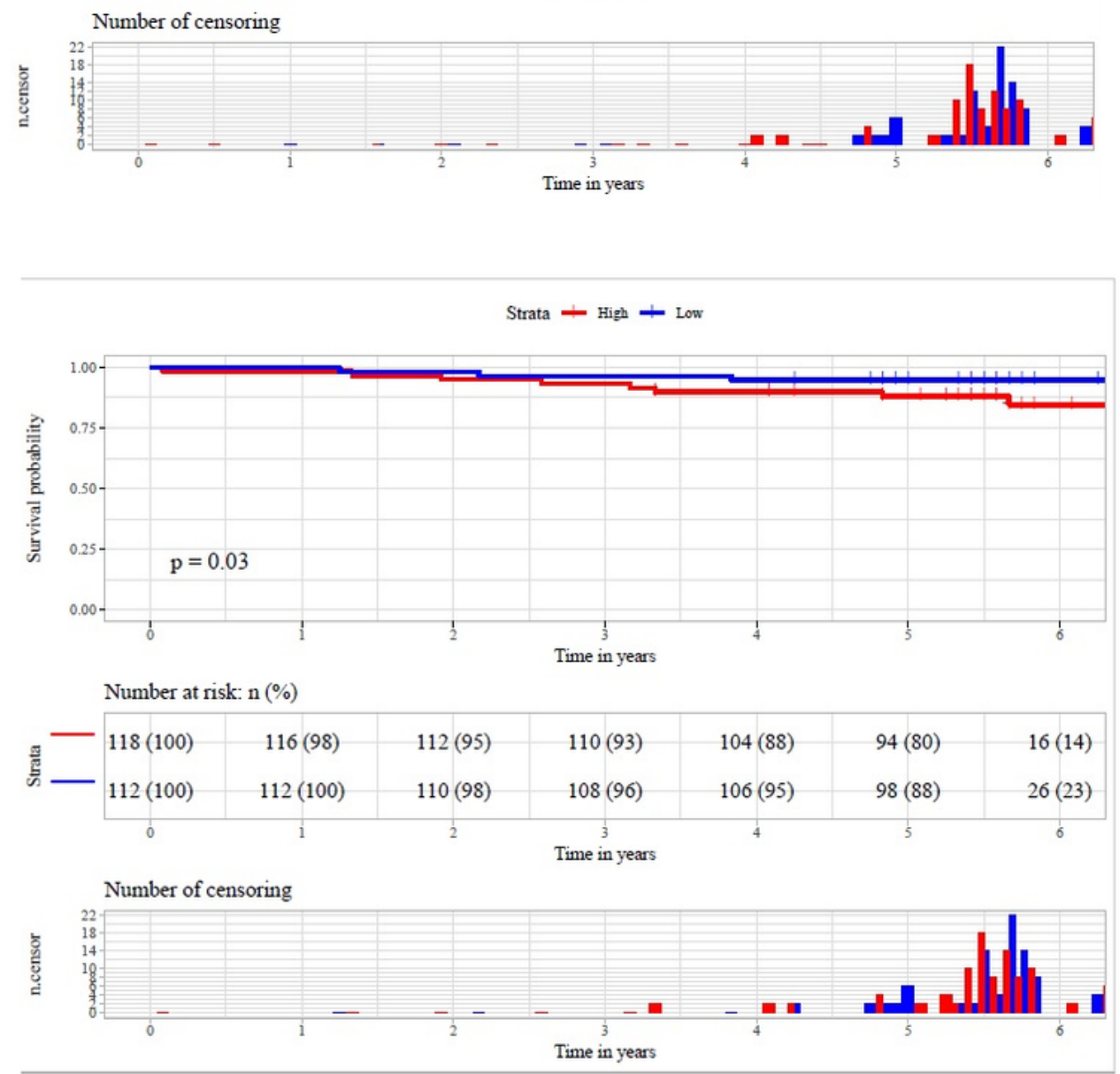

\section{Figure 1}

Kaplan-Meier survival analysis of DFS(A) and OS(B) according to low and high groups. 


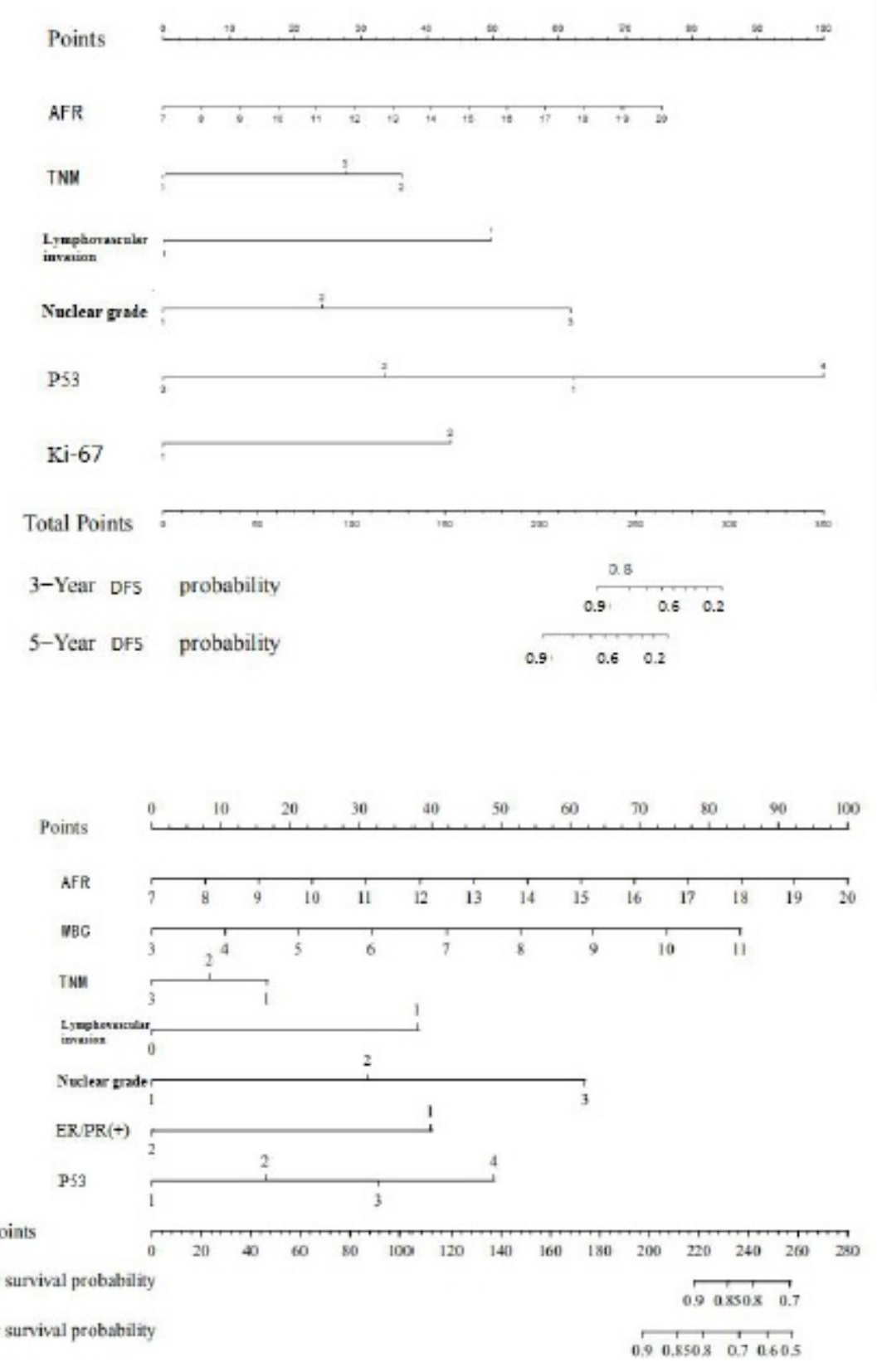

\section{Figure 2}

Nomogram model predicting 3- and 5-year DFS(A) and OS(B) in breast invasive ductal carcinoma patients. The nomogram was used summing the points identified on the points scale for each variable. The total points projected on the bottom scales indicate the probability of 3- and 5-year survival.

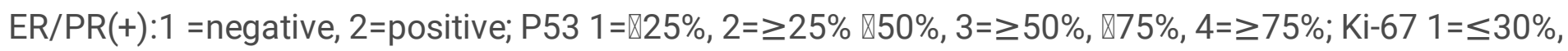
$2=\rrbracket 30 \%$, Lymphovascular invasion $0=\mathrm{NO}, 1=Y$ es. 


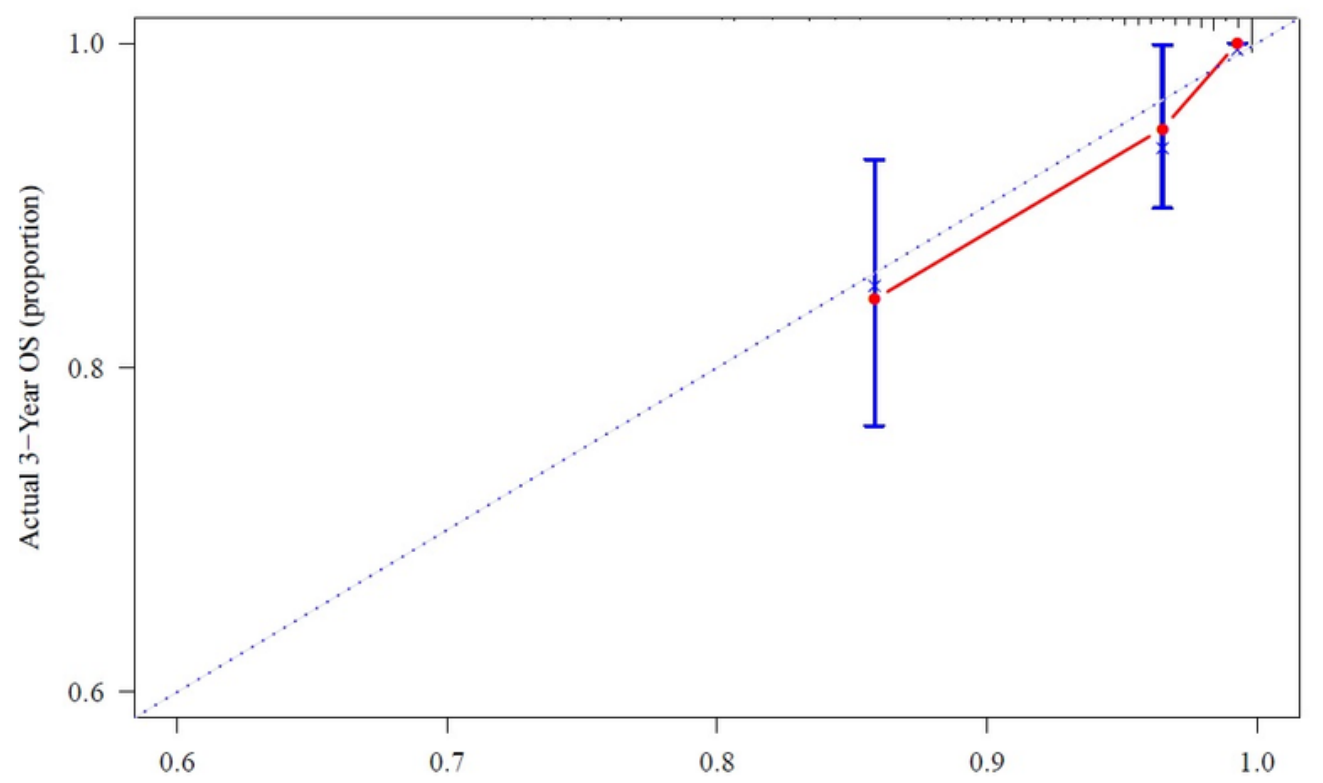

$\mathrm{n}=228 \mathrm{~d}=34 \mathrm{p}=6,70$ subjects per group Nomogram-Predicted Probability of 3 -Year OS - resampling optimism added, $\mathrm{B}=1000$ Gray: ideal

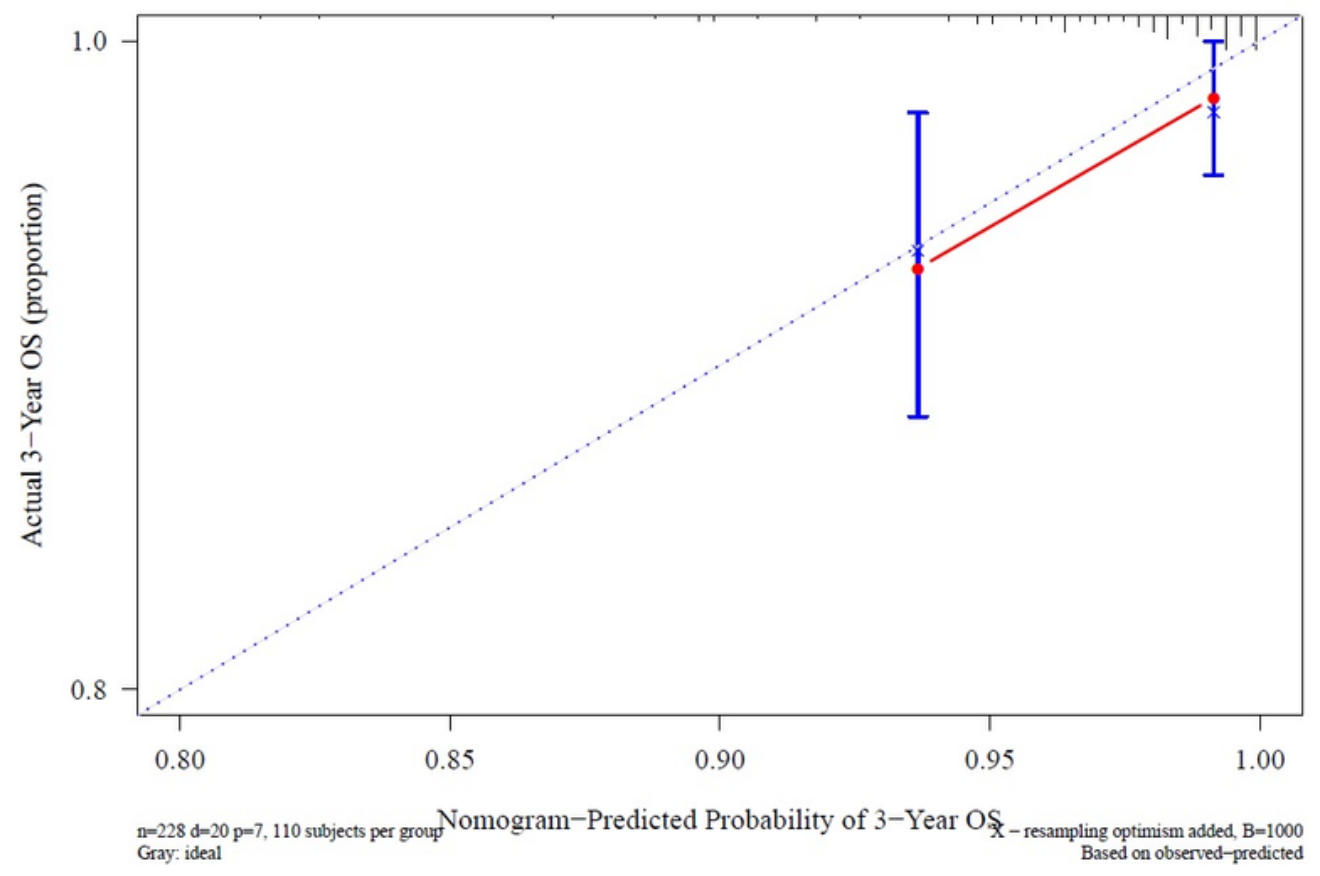

\section{Figure 3}

The curves for predicting patient DFS(A) and OS(B) at three years. Nomogram model-predicted OS is plotted on the $\mathrm{x}$-axis; actual OS is plotted on the $y$-axis. Closer alignment with the diagonal line represents a better estimation. 

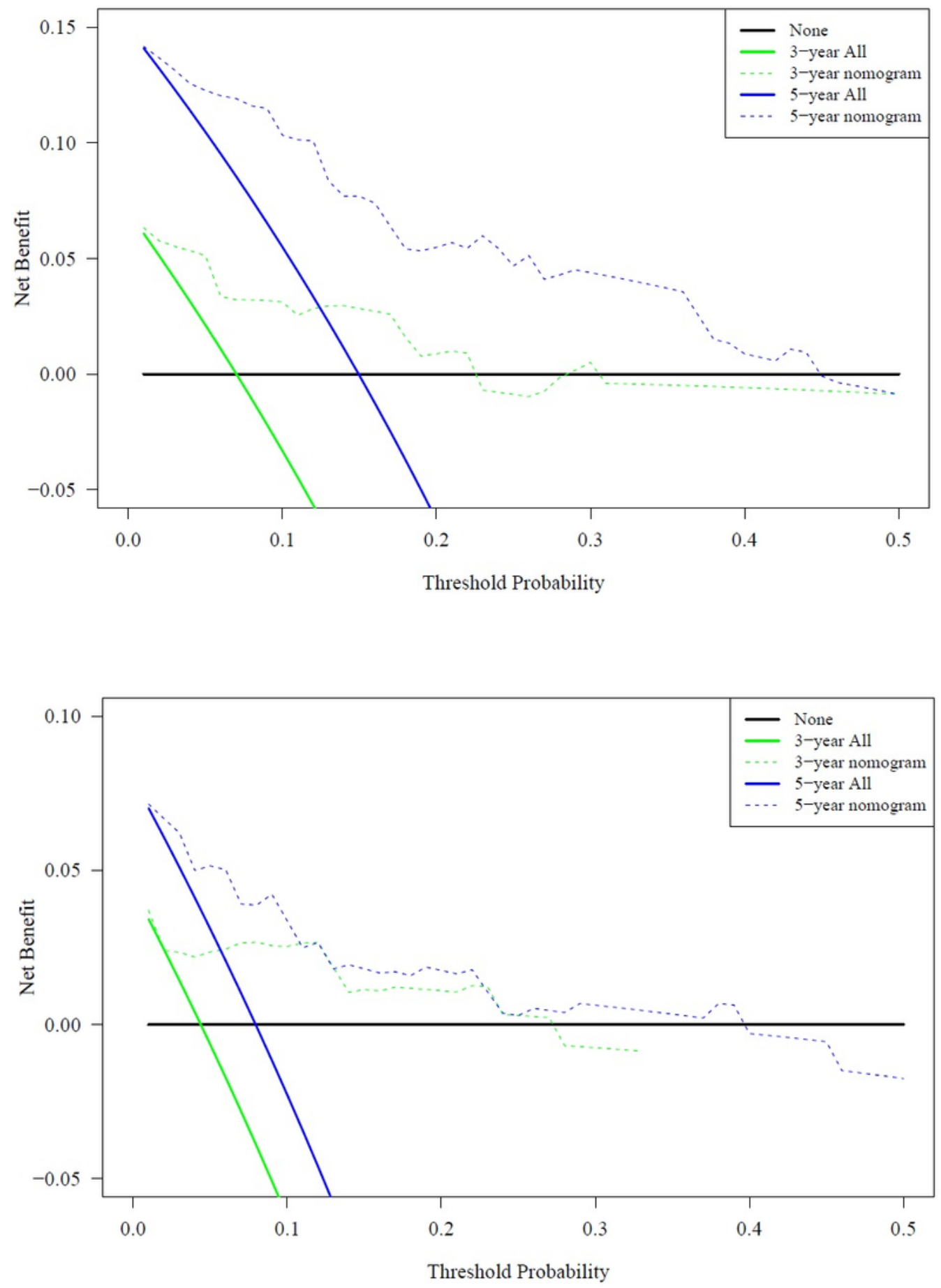

\section{Figure 4}

Decision curve analysis for the two nomograms in the population,DFS(A) and OS(B). The $y$-axis measures the net benefit. The dotted lines ( green and blue) represent the nomogram. The solid lines (green and blue) represent the assumption that all patients have 3-, or 5-year survival and DFS, respectively. The thin black line represents the assumption that no patients have 3-, or 5-year survival and 
DFS. The net benefit was calculated by subtracting the proportion of all patients who are false positive from the proportion who are true positive. 